\title{
Etude expérimentale des débits de vaporisation dans les poches de cavitation
}

\author{
par B. Stutz et J.L. Reboud \\ LEGI, Institut de mécanique de Grenoble
}

\section{I INTRODUCTION}

Le phénomène de cavitation consiste en une succession de processus de vaporisation et de condensation d'un fluide sans apport de chaleur extérieur. Les transferts de chaleur latente, nécessaires aux changements de phase liquide-vapeur, sont effectués avec le fluide environnant. La vaporisation du liquide induit un refroidissement local, et de ce fait un abaissement de la pression de vapeur saturante. Ce phénomène, négligeable dans le cas de l'eau froide, peut limiter de manière sensible le développement des poches de cavitation dans le cas de fluides dit thermosensibles. Il sera d'autant plus important que la quantité de liquide vaporisé est grande. L'estimation de tels effets nécessite de ce fait une connaissance des flux de vaporisation.

La majorité des travaux expérimentaux consacrés à l'estimation des débits au sein des poches de cavitation reposent sur une même technique d'investigation : la ventilation (injection de gaz non condensable). Le débit volume de vapeur au sein des poches de cavitation est supposé égal au débit volume de gaz non condensable nécessaire à l'entretien d'une poche ventilée de même forme et de même longueur. Les lois de comportement disponibles dans la littérature et établies à l'aide de cette méthode sur des profils bidimensionnels montrent des coefficients de débits compris entre $2.10^{3}$ et $13.10^{3}$, sensibles selon les profils à la vitesse de l'écoulement et aux tailles de poche [1]. Cette méthode repose sur une hypothèse forte de similitude de comportement des écoulements cavitants et ventilés. Elle constitue une application du modèle physique de l'entraînement proposé par Acosta et Perkin [2], concernant les mécanismes d'évacuation de la phase gazeuse en fermeture de poche. Les effets d'entraînement des particules gazeuses dans le sillage des poches sont considérés prédominants par rapport aux effets de condensation propres aux écoulements cavitants. Quel que soit le type de géométrie étudié, (profil sphérique, disque, ogive, Venturi, hydrofoil), le comportement des poches ventilées apparaît en effet très voisin de celui de la cavitation naturelle notamment en ce qui concerne les lois de similitude de longueur de poche par rapport au nombre de cavitation (basé sur la pression au sein des cavités), la forme extérieure des parties amont des poches, l'évolution de la pression réduite moyenne au voisinage de la paroi ainsi que les pertes de charges générées [3], [4], [5].

\begin{tabular}{|c|c|c|c|c|}
\hline \multicolumn{5}{|c|}{ NOMENCLATURE } \\
\hline \multirow{3}{*}{$\begin{array}{l}\mathrm{B} \\
\mathrm{C}_{\mathrm{pl}}\end{array}$} & \multirow{3}{*}{$\begin{array}{l}\text { Largeur de la veine } \\
\text { Chaleur massique (ou spécifique) } \\
\text { du liquide }\end{array}$} & \multirow{3}{*}{$\begin{array}{r}\mathrm{m} \\
\mathrm{J} / \mathrm{kg} /{ }^{\circ} \mathrm{KC}_{\mathrm{q}}\end{array}$} & \multirow[t]{2}{*}{$T_{\text {amont }}$} & \multirow{2}{*}{$\begin{array}{l}\text { Température mesurée au niveau d'une section } \\
\text { de référence amont }\end{array}$} \\
\hline & & & & \\
\hline & & & TV & Taux de vide (donné en \%) \\
\hline $\mathrm{C}_{\mathrm{q}}$ & Coefficient de débit $C_{q}=Q / B \cdot L \cdot V_{r e f}$ & \multirow{3}{*}{$\mathrm{m}$} & $\mathrm{V}$ & Vitesse \\
\hline $\mathrm{L}$ & Longueur de poche & & \multirow[t]{2}{*}{$\mathrm{V}_{\text {amont }}$} & \multirow{2}{*}{$\begin{array}{l}\text { Vitesse débitante au niveau d'une section } \\
\text { de référence amont }\end{array}$} \\
\hline$L$ & Chaleur latente de vaporisation & & & \\
\hline$P$ & Pression $\left(\mathrm{N} / \mathrm{m}^{2}\right)$ & & $V_{\text {ref }}$ & Vitesse débitante de référence \\
\hline$P_{c a v}$ & Priession dans la cavité $\approx \mathrm{P}_{\mathrm{v}}\left(\mathrm{T}_{\text {cav }}\right)$ & $\mathrm{N} / \mathrm{m}^{2}$ & $\mathrm{X}$ & Abscisse \\
\hline $\mathrm{P}_{\mathrm{o} 2}$ & Pression d'oxygène dissous dans l'eau & ppm & $\mathrm{Y}$ & Ordonnée verticale \\
\hline $\mathrm{P}_{\mathrm{ref}}$ & Pression statique de référence & $\mathrm{N} / \mathrm{m}^{2}$ & $\alpha$ & Incidence \\
\hline$P_{\text {momt }}$ & Pression statique au niveau d'une section & $\mathrm{N} / \mathrm{m}^{2}$ & $\rho_{\mathrm{c}}$ & Masse volumique du gaz ou de la vapeur \\
\hline & de référence amont & & $\rho_{\mathrm{L}}$ & Masse volumique du liquide \\
\hline Q & Débit volume & $\mathrm{m}^{3} / \mathrm{s}$ & $\sigma$ & \multirow{2}{*}{$\begin{array}{l}\text { Nombre de cavitation basé sur la pression au sein } \\
\text { des cavités } \sigma=\left(\mathrm{P}_{\mathrm{amont}}-\mathrm{P}_{\mathrm{cav}}\right) / 1 / 2 \rho \mathrm{V}_{\mathrm{mont}}{ }^{2}\end{array}$} \\
\hline $\mathrm{T}$ & Température & ${ }^{\circ} \mathrm{C}$ & & \\
\hline
\end{tabular}




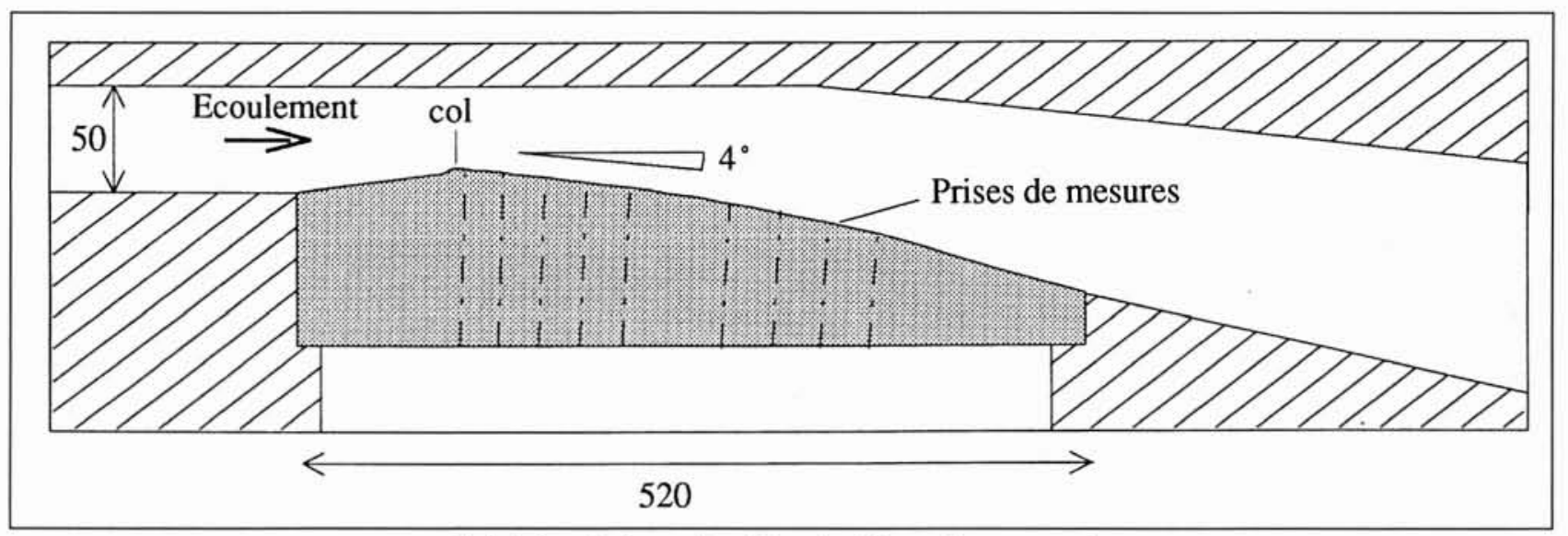

1. Schéma de la section d'essais (dimensions en mm).

Le présent article a pour objet de compléter ces travaux par une étude comparative des écoulements diphasiques au sein des poches ventilées et cavitantes à l'eau. Les champs de vitesse et de taux de vide seront déterminés expérimentalement par le biais de mesures locales par bisonde optique. Leur intégration donnera accès au débit de la phase gazeuse. Ils seront ensuite confrontés aux résultats obtenus dans le cas d'une poche de cavitation au réfrigérant $\mathrm{R} 114$, fluide thermosensible.

\section{II — DISPOSITIF EXPÉRIMENTAL}

\subsection{Les veines d'essais}

Pour des questions d'accessibilité, les essais sont menés sur des veines de type Venturi. Deux boucles d'essais, construites sur le principe de circuits hydrauliques fermés, sont utilisées : la première, à l'eau, comporte une cuve à surface libre permettant de pressuriser le circuit ainsi que de résorber les bulles de gaz contenues dans l'écoulement. La teneur en oxygène dissous du liquide de circulation est contrôlée par une sonde à oxygène de type ampérométrique à membrane située en aval de la veine d'essais. Les expérimentations sont réalisées avec un taux d'oxygène dissous à l'équilibre au niveau de la cuve aval. Cette condition assure une grande stabilité du point de fonctionnement, ainsi que la présence d'une très faible densité de germes occlus. La deuxième boucle d'essais au Réfrigérant R114 ne comporte pas de surface libre. La régulation de la température du fluide est assurée par le biais d'un échangeur monté en bypass sur le circuit, la pressurisation de la boucle par une vessie gonflée à l'azote. Ce système assure en principe l'absence de gaz dissous dans le fluide. Un contrôle de la teneur en germes du liquide de circulation n'a révélé aucune présence de particule avant ensemencement spécifique pour la vélocimétrie laser [6]. Les régulations des conditions de fonctionnement sont assurées par le biais d'un débitmètre ( $D Q / Q= \pm 0,3 \%)$, d'un capteur de pression piezo-résistif $(\Delta \mathrm{P} / \mathrm{P}= \pm 0,1 \%)$ et d'une sonde de température platine $100 \Omega\left(\Delta \mathrm{T}<0,1^{\circ} \mathrm{C}\right)$ situés en amont de la veine d'essais.

Les essais sont menés sur une même configuration de veine, à parois latérales parallèles et sections transversales rectangulaires (section amont : hauteur $50 \mathrm{~mm}$; largeur $44 \mathrm{~mm}$ ). La convergence puis la divergence du conduit hydraulique sont assurées par les faces supérieures et inférieures. Leur géométrie a été conçue de façon à reproduire, en subcavitant, les gradients de pression présents sur l'extrados d'un inducteur de pompe. La section au col est de 43,7 × $44 \mathrm{~mm}$, la divergence du conduit voisine de $4^{\circ}$ (Figure 1). Une arête vive, localisée au col de la veine d'essais, permet de fixer les départs de poches. Les veines d'essais équipées de la présente géométrie sont caractérisées par un blocage cavitant apparaissant pour des poches de tailles supérieures à $12 \mathrm{~cm}$. Les vitesses débitantes au col sont comprises entre 5 et $20 \mathrm{~m} / \mathrm{s}$ à l'eau et 16 à $45 \mathrm{~m} / \mathrm{s}$ au Réfrigérant R114. Les températures, voisines de $20^{\circ} \mathrm{C}$ à l'eau, peuvent être régulées de 20 à $40^{\circ} \mathrm{C}$ au Réfrigérant R114.

Un profil spécifique a été doté d'une fente d'injection d'air en aval du col de la veine [7]. Son implantation sur la veine à l'eau permet d'étudier les poches ventilées. L'air injecté est directement prélevé au niveau de la cuve aval, ce qui assure une grande stabilité des points de fonctionnement. Le débit d'air injecté, régulé par une vanne pointeau, est mesuré par un débitmètre à flotteur, calibré de façon à travailler à pression atmosphérique. La pression d'injection est contrôlée en sortie du débitmètre ainsi qu'au sein des cavités au moyen de capteurs piézorésistifs Druck. La pression statique de l'écoulement est maintenue suffisamment haute pour prévenir tout risque de vaporisation du liquide lors de l'étude des poches ventilées. Le dispositif actuel d'injection d'air restreint le domaine d'étude des poches ventilées à des vitesses de $14 \mathrm{~m} / \mathrm{s}$.

\subsection{Instrumentation}

\subsubsection{Mesure au sein des écoulements monophasés liquides}

Des mesures de pression et de température sont réalisées de manière classique sur la paroi inférieure de la veine d'essais par le biais de capteurs de pression $\left(\Delta \mathrm{P} / \mathrm{P}_{\max }= \pm 0,1 \%\right)$ et de thermocouples de type $\mathrm{T}$ à faible pouvoir thermoélectrique $(\Delta \mathrm{T}=$ $\pm 0,2^{\circ} \mathrm{C}$ ). Les formes extérieures des poches de cavitation sont déterminées au moyen d'un dispositif d'analyse d'image développé au CREMHyG [8], dont le principe repose sur la réflexion d'une lumière incidente sur l'interface diphasique constituant la frontière supérieure des poches ou sur le fond de la veine.

\subsubsection{Mesures au sein des écoulements diphasiques}

Un dispositif de mesure locale par bisonde optique a été développé afin de pouvoir étudier les écoulements diphasiques au sein des poches de cavitation [9]. Ce type de capteur, dont les 


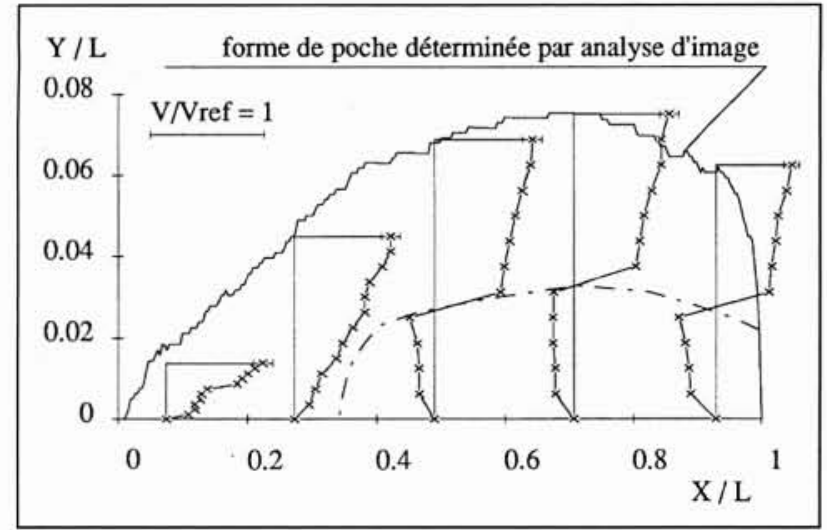

2. Evolution de la vitesse tangentielle au sein d'une poche de cavitation à l'eau.

interactions avec l'écoulement s'apparentent à celles rencontrées lors de l'utilisation de sondes de type résistive, est couramment utilisé pour l'étude des écoulements diphasiques à faible vitesse $(<1 \mathrm{~m} / \mathrm{s})$, constitués de bulles de dimensions élevées vis-à-vis des parties sensibles des sondes [10]. La particularité des écoulements étudiés, caractérisés par des vitesses pouvant atteindre $35 \mathrm{~m} / \mathrm{s}$, des changements de phases brutaux et des tailles de particules vapeur extrêmement variées, limitent l'éventail des traitements du signal aux techniques de discrimination par un seuil unique pour l'estimation du taux de vide et d'inter-corrélation des signaux pour l'obtention de la vitesse locale moyenne. L'étalonnage et la validation des traitements mis en œuvre ont fait l'objet de nombreuses campagnes de mesures [11]. Elles ont permis d'estimer les incertitudes relatives du capteur quant à l'estimation du taux de vide à $\Delta T V / T V$ $= \pm 15 \%$ et à la mesure de la vitesse locale moyenne à :

$\Delta \overline{\mathrm{V}} / \overline{\mathrm{V}} \approx \pm 10^{2} \sqrt{2+0,56 . \mathrm{V}^{2}}$

Les débits volumes des phases liquide et vapeur sont estimés au droit de chacune des prises de mesures par intégration des mesures locales. Les bornes d'intégration sont données par la paroi inférieure de la veine et l'interface des poches de cavitation, correspondant à un taux de vide de $1 \%$. Les calculs reposent sur une hypothèse d'écoulement diphasique bidimensionnel au sein des poches de cavitation. Par ailleurs, les couches limites latérales et le glissement entre phases sont supposés négligeables. Les débits volumes de la phase k seront exprimés sous la forme adimensionnelle d'un coefficient de débit : $\mathrm{C}_{\mathrm{q}}=\mathrm{Q}_{\mathrm{x}} / \mathrm{B} \cdot \mathrm{L} \cdot \mathrm{V}_{\mathrm{ref}}$

\section{III — RÉSULTATS EXPÉRIMENTAUX}

Quel que soit le type d'essais, les longueurs de poches sont fonction du nombre de cavitation $\sigma$ calculé à partir de la pression au sein de la partie amont des cavités. Par la suite, les résultats seront exprimés par rapport à la vitesse de référence estimée à l'interface des cavités en négligeant les pertes visqueuses amont:

$$
\mathrm{V}_{\mathrm{ref}}=\mathrm{V}_{\mathrm{amont}} \cdot \sqrt{1+\sigma}
$$

\subsection{Poches de cavitation à l'eau}

Les résultats présentés sont caractéristiques des écoulements diphasiques observés au sein des poches de cavitation à l'eau.

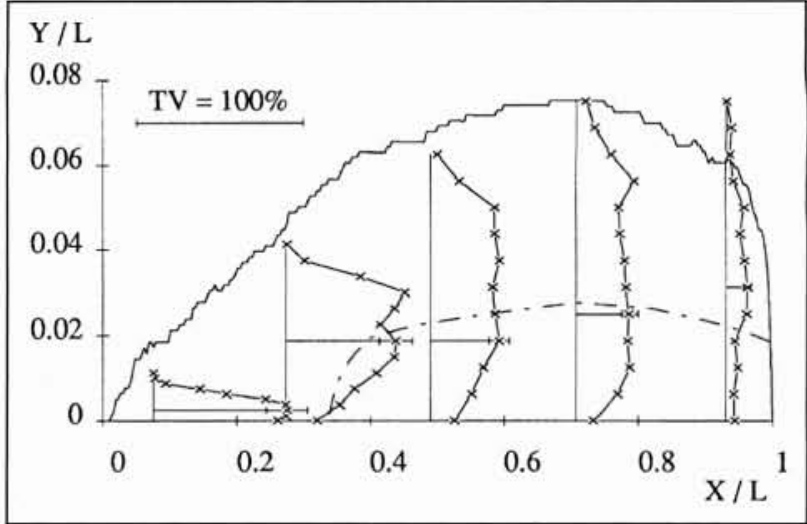

3. Evolution du taux de vide au sein d'une poche de cavitation à l'eau.

Ils ont été obtenus dans les configurations d'écoulement suivantes : $\mathrm{V}_{\mathrm{r} r}=14 \mathrm{~m} / \mathrm{s}, \mathrm{L}=80 \mathrm{~mm}, \sigma=0,68$.

\subsubsection{Ecoulements diphasiques au sein des poches de cavitation à l'eau (Figures 2 et 3)}

La vitesse à l'interface des poches apparaît constante et voisine de 0,85 fois la vitesse de référence sur les $2 / 3$ amont des cavités, montrant un déficit de vitesse, probablement dû à la convection du profil de couche limite se développant en amont du bord d'attaque. Elle diminue ensuite régulièrement en aval dans la zone de fermeture. Une recirculation marquée apparaît au voisinage de la paroi sur les deux tiers aval de la poche. Elle est caractérisée par une vitesse voisine de 0,2 fois la vitesse de référence et une épaisseur voisine de $40 \%$ de la hauteur totale de la poche. Cet écoulement adverse apparaît spécifique au régime cavitant, aucun comportement semblable n'ayant pu être mis en évidence en l'absence de cavité sur ce profil.

Contrairement à l'hypothèse antérieure assimilant les poches de cavitation à des cavités de vapeur pure [12], [13], les profils de taux de vide montrent la nature diphasique des écoulements internes dont la teneur en vapeur évolue régulièrement tout au long du profil. Le taux de vide atteint une valeur maximale voisine de $80 \%$ en début et à mi-hauteur de poche. Il diminue ensuite régulièrement tout en s'homogénéisant sur toute la hauteur jusqu'à la zone de fermeture. L'écoulement adverse est composé d'une part non négligeable de vapeur similaire à celle mesurée au sein de l'écoulement diphasique principal de la poche. Sa croissance tout au long de sa remontée semble être la conséquence d'un processus de vaporisation de l'écoulement adverse.

\subsubsection{Estimation des débits au sein des poches (Fig. 6)}

L'intégration des mesures locales par bisonde optique permet de suivre les évolutions des débits volumes des phases présentes au sein des poches de cavitation. L'évolution du débit volume de vapeur est caractérisé par une forte croissance sur le premier tiers de la poche (section 1 et 2 ), conséquence d'une vaporisation intense du liquide, puis diminue ensuite régulièrement jusqu'à la zone de fermeture (section 5). Sa proportion ne dépasse pas $50 \%$ du débit volume total au sein d'une même section. Le débit maximum de vapeur au sein des poches équivaut à un coefficient de débit de l'ordre de $\mathrm{C}_{\mathrm{q}}=8,5 \cdot 10^{3}$. 


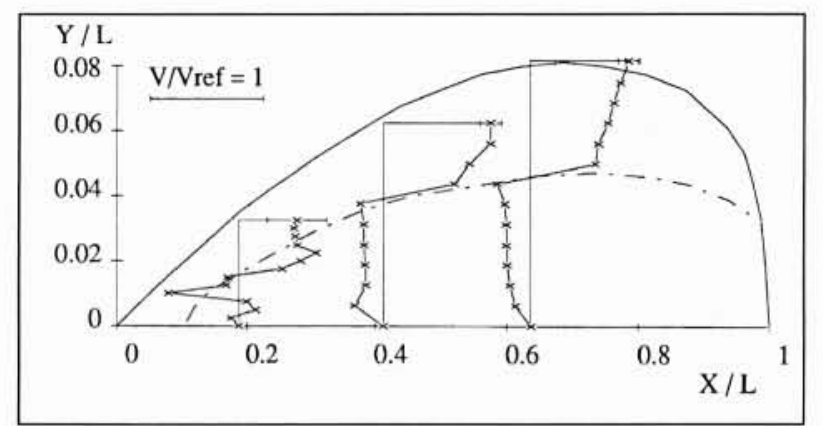

4. Evolution de la vitesse tangentielle au sein d'une poche ventilée à l'eau.

\subsection{Poches ventilées}

L'étude des écoulements diphasiques au sein des poches ventilées a été réalisée dans la configuration d'écoulement suivante : $\mathrm{V}_{\mathrm{re}}=14 \mathrm{~m} / \mathrm{s}$ et $\mathrm{L}=80 \mathrm{~mm}$. Le "nombre de cavitation" $\sigma$, mesuré à l'aide de la mesure de pression de gaz dans la poche, équivaut à celui obtenu auparavant : 0,68. L'encombrement du dispositif expérimental d'injection d'air limite à trois le nombre d'axes de mesure.

\subsubsection{Ecoulements diphasiques au sein des poches ven- tilées (Figures 4 et 5 )}

La partie amont de l'écoulement interne de la poche ventilée s'apparente à un écoulement cisaillé stratifié : elle est composée dans sa partie basse d'une structure diphasique dispersée à faible teneur en gaz (Taux de vide inférieur à $20 \%$ ). Les difficultés de mesure de la vitesse dans cette région traduisent l'instabilité de l'écoulement local, s'apparentant à une structure tourbillonnaire. Sa partie supérieure est quant à elle caractéristique d'un jet de gaz (taux de vide voisin de $70 \%$; vitesse uniforme voisine de $0,5 \mathrm{~V}_{\text {ret }}$; taille de corde moyenne supérieure à six fois la hauteur de poche). Les deux tiers aval de la poche de gaz sont caractérisés par des profils de taux de vide relativement homogènes voisins de $40 \%$ sur toute la hauteur. Ils mettent en évidence l'action de l'écoulement adverse sur le fractionnement du jet gazeux issu de la fente d'injection d'air. L'écoulement adverse, beaucoup plus développé que dans le cas de la cavitation à l'eau, occupe plus de $50 \%$ de la hauteur de poche.

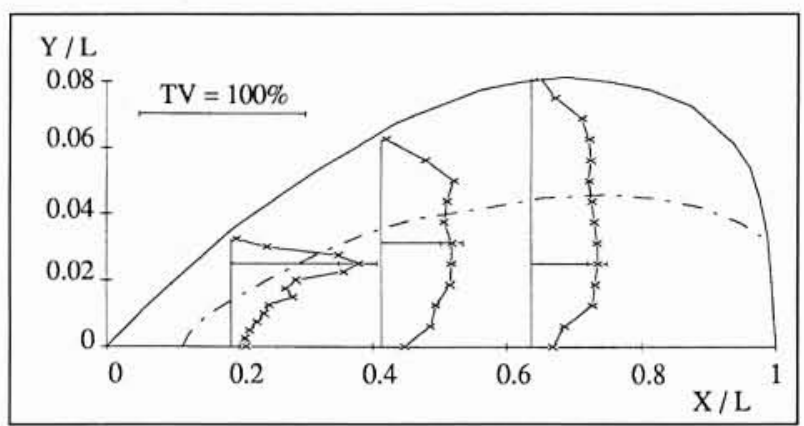

5. Evolution du taux de vide au sein d'une poche ventilée à l'eau.

\subsubsection{Analyse comparative des débits de vapeur}

Le débit d'air injecté, nécessaire à la génération de la poche ventilée, équivaut à un coefficient de débit de $\mathrm{C}_{\mathrm{q}}=3,04 \cdot 10^{-3} \pm$ 0,3 . Les faibles gradients de pression au sein des poches permettent de faire l'hypothèse d'un débit volume d'air constant entre la fente d'injection et les trois plans de mesure.

Les débits volume d'air reconstitués à l'aide de la bisonde optique sont respectivement de $\mathrm{C}_{\mathrm{q} 1}=3,65.10^{3} \pm 0,8 ; \mathrm{C}_{\mathrm{q} 2}=$ $3,14.10^{3} \pm 0,5 ; \mathrm{C}_{4^{3}}=2,84.10^{3} \pm 0,5$. L'incertitude importante des estimations du débit au niveau de la première prise de mesure provient essentiellement des difficultés de mesure de la vitesse en un tel plan (fluctuations élevées de l'écoulement au voisinage du détachement des poches dans le cas de la ventilation). L'ensemble des mesures montre cependant une bonne similarité des résultats se recoupant sur leurs domaines d'incertitude et restant voisins des débits injectés. Cela confirme la pertinence de la méthode d'estimation du débit à l'aide de mesures locales par bisonde optique.

Les évolutions des débits volumes de la phase gazeuse sont reportées figure 6. Des différences importantes apparaissent entre les poches ventilées et cavitantes se développant sur les deux profils. Elles ont conduit à la réalisation d'essais cavitants complémentaires sur le profil muni du dispositif de ventilation en obstruant la fente d'injection d'air. Les débits de vapeur, mesurés apparaissent alors relativement voisins de ceux obtenus dans le cas de la ventilation. La limitation des flux de vaporisation sur le profil muni d'une fente d'injection d'air est à mettre en parallèle avec le développement conséquent de l'écoulement

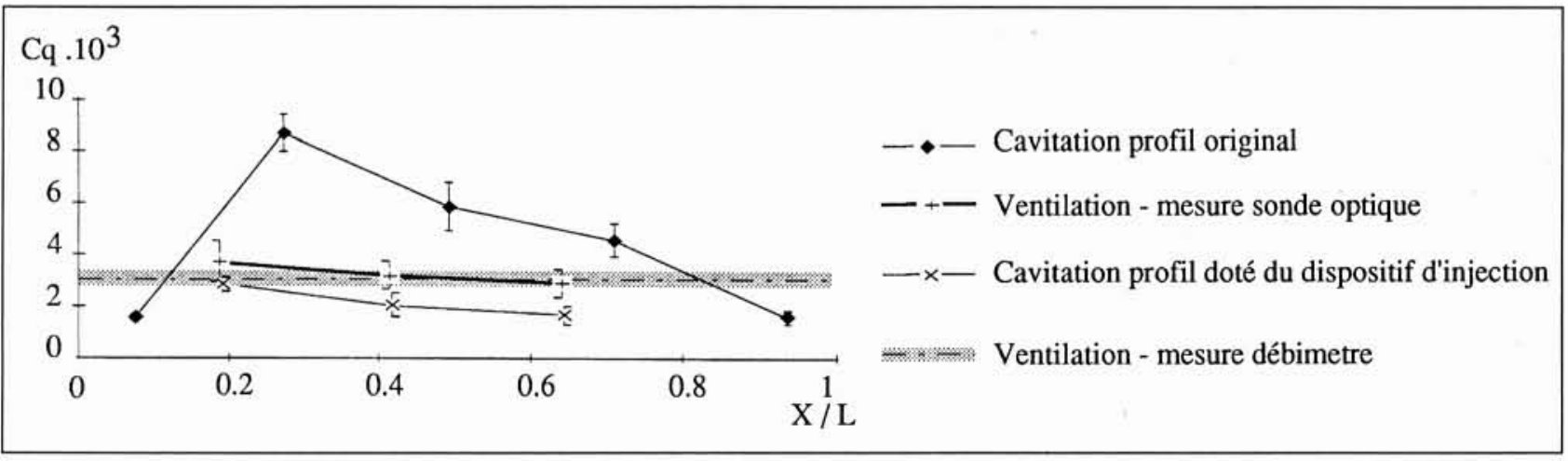

6. Evolution des débits volumes de la phase gazeuse au sein des poches ventilées et cavitantes. 
adverse (de taille similaire à celle observée au sein des poches ventilées) et dont la croissance apparaît limiter le flux de vaporisation au sein des poches de cavitation.

Les écoulements diphasiques au sein des poches de cavitation apparaissent ainsi sensibles à la géométrie fine des profils : les faibles différences de rugosité apparaissent en effet suffisantes pour modifier de manière significative le développement de l'écoulement adverse au voisinage de la paroi et par conséquent le taux de la vaporisation du fluide. (rugosité du profil original : $\mathrm{R}_{\mathrm{u}}=0,8$; rugosité du profil doté d'un dispositif d'injection d'air : $R_{a}=0,15$ )

\subsection{Poches de cavitation au réfrigérant $\mathbf{R 1 1 4}$}

Les plages de fonctionnement des veines d'essais à l'eau et au réfrigérant R114 limitent les études comparatives des écoulements cavitants de la présente géométrie à des conditions d'isovitesse. La configuration d'écoulement retenue est la suivante : $\mathrm{V}_{\mathrm{rac}}=18,8 \mathrm{~m} / \mathrm{s}, \mathrm{L}=80 \mathrm{~mm}$.

\subsubsection{Caractéristique des écoulements cavitants au réfrigérant $R 114$}

$\mathrm{Si}$, pour des conditions de pression données en entrée de la veine d'essais, les effets thermodynamiques limitent de façon sensible le développement des poches de cavitation au réfrigérant R114 par rapport à celui observé à l'eau, les lois d'évolution des tailles de poches en fonction du nombre de cavitation local $\sigma_{\mathrm{cav}}$ apparaissent similaires [13]. De même, les géométries des poches se superposent relativement bien. Les évolutions de la température mesurée sur la paroi inférieure de la veine au réfrigérant R114 sont montrées figure 7. Le refroidissement apparaît varier linéairement d'amont en aval [6]. Il apparaît maximum en début de poche pour s'annuler au niveau de la zone de fermeture. L'allongement des poches induit un élargissement de la zone affectée thermiquement, alors que l'échauffement du liquide et l'accroissement des temps de transit des particules de fluides au sein des cavités, fonction de la vitesse de l'écoulement, tend à accentuer l'intensité de ces effets. En faisant l'hypothèse d'une vaporisation adiabatique du liquide au sein des poches et d'une homogénéité du milieu diphasique selon la normale au profil, il est possible d'estimer la valeur moyenne du taux de vide au niveau de chaque section de mesure à partir des mesures de température :

$$
\mathrm{TV}=\frac{\Delta \mathrm{T}}{\Delta \mathrm{T}+\Delta \mathrm{T}_{\mathrm{car}}} \operatorname{avec} \Delta T_{c a r}=\frac{\rho_{G} \mathcal{L}}{\rho_{L} C_{p L}}
$$

\subsubsection{Analyse des signaux temporels (Figure 8)}

Les signaux délivrés par la bisonde optique lors de l'étude des écoulements diphasiques au sein des poches de cavitation et des poches ventilées à l'eau sont caractéristiques de perçage de particules. Il en va autrement dans le cas des écoulements au réfrigérant R114, pour lesquels ils apparaissent plus chaotiques et semblent le fait de réflexions multiples de la lumière incidente sur une population de bulles contournant les sondes sans être percées. L'application de modèle de fractionnement classique basé sur une loi de type Weber critique tel que celui proposé par Sevik et Park [14], confirment l'hypothèse d'un fractionnement accru des structures gazeuses dans le cas du réfrigérant R114: les tailles limites de structures gazeuses y apparaissent en effet 5 fois plus faibles qu'à l'eau. Cet effet de tension superficielle pourrait être accentué du fait de la cinétique thermique sensible dans le cas de l'utilisation du réfrigérant R114 [15]. La tenue mécanique des capteurs ne permet malheureusement pas d'envisager une miniaturisation plus importante des têtes de sondes propice à l'obtention de meilleures mesures. Pour pallier cette déficience, une nouvelle technique d'estimation du taux de vide local basée sur une analyse dimensionnelle des signaux a été développée. Son principe repose sur une mesure de l'indice de réfraction moyen du milieu environnant les sondes. L'absence d'étalonnage actuel ne permet pas d'estimer l'étendue de sa validité. Les résultats obtenus ne seront donc donnés qu'à titre indicatif. A l'inverse, la mesure de la vitesse locale par inter-corrélation des signaux ne pâtit pas de la dégradation des signaux : son principe est en effet basé sur une estimation du temps de transit le plus probable de perturbations d'une sonde à l'autre.

\subsubsection{Ecoulements diphasiques au sein des poches de cavitation au réfrigérant R114 (Figures 9 et 10)}

Les évolutions de la vitesse au sein des poches de cavitation au réfrigérant R114 sont assez semblables à celles obtenues à l'eau. L'écoulement adverse présent dans la partie basse des

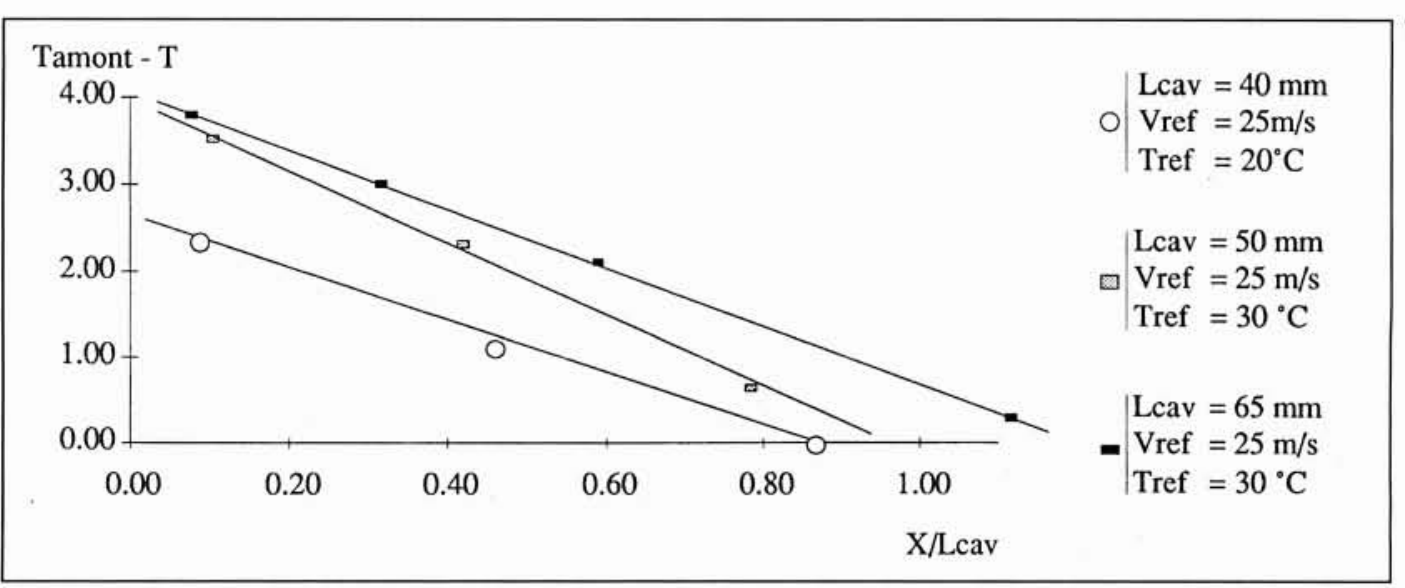

7. Evolution de la température au sein des poches de cavitation au réfrigérant $\mathbf{R} 114$. 


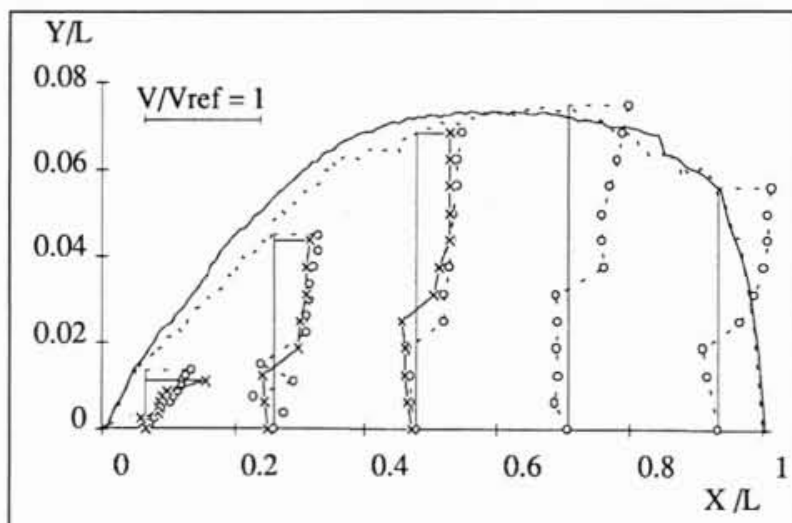

Vref $=18.8 \mathrm{~m} / \mathrm{s} ;$ Tamont $=20^{\circ} \mathrm{C} ; \mathrm{L}=80 \mathrm{~mm}$

$$
\because \quad \text { Eau } \times \text { Réfrigérant R114 }
$$

\section{Evolution comparative de la vitesse au sein des poches} de cavitation à l'eau et au réfrigérant $R 114$.

poches y apparaît cependant plus développé. Les effets du Reynolds, quatre fois plus élevés qu'à l'eau, ainsi que les phénomènes de cinétique thermique générée par les effets thermodynamiques peuvent en être la cause. L'interaction occasionnelle de l'écoulement rentrant avec l'interface diphasique est à l'origine d'arrachements sporadiques des poches de cavitation observés sur les films rapides réalisés par la SEP, mais n'apparaît pas suffisante pour induire un comportement instationnaire périodique de type auto-oscillant.

Les évolutions du taux de vide estimées par bisonde optique au sein des poches de cavitation au réfrigérant R114 sont similaires à celles observées à l'eau pour les profils 2 et 3 situés respectivement à $\mathrm{X} / \mathrm{L}=0,26$ et $\mathrm{X} / \mathrm{L}=0,48$. A l'inverse, ils divergent au niveau de la partie amont des poches : la proportion de vapeur atteint $40 \%$ dans le cas du réfrigérant R114 alors qu'elle est d'environ $80 \%$ dans le cas de l'eau. Cette restriction du taux de vide est à mettre en parallèle avec la taille de l'écoulement adverse, plus développée dans le cas du réfrigérant R114, ainsi qu'aux phénomènes de cinétique thermique plus développés dans le cas de fluides thermosensibles. Les estimations du taux de vide moyen déterminées à partir des mesures de température en paroi sont voisines du taux de vide maximum mesuré à l'eau au niveau de chacune des sections. Elles vont à l'en-

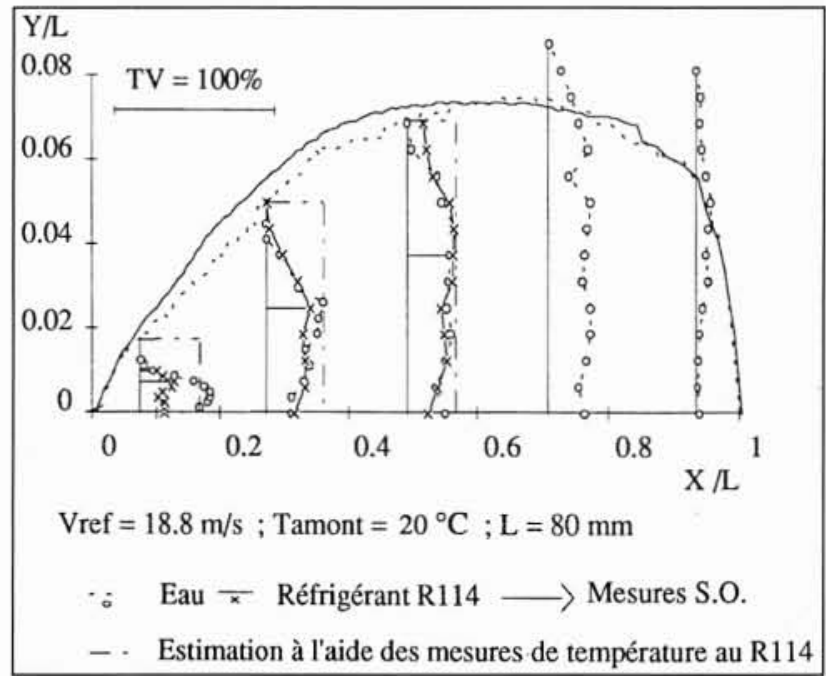

10. Evolution comparative du taux de vide au sein de poches de cavitation à l'eau et au réfrigérant R114.

contre de l'évolution du taux de vide estimé par bisonde optique sur la partie amont des poches, et appellent de ce fait à la réalisation d'essais complémentaires.

Les débits volumes de vapeur au sein des poches de cavitation au réfrigérant $\mathrm{R} 114$ apparaissent du même ordre de grandeur que ceux obtenus à l'eau. Leurs valeurs maximales équivalent à un coefficient de débit de l'ordre de $\mathrm{C}_{\mathrm{q}}=8 \cdot 10^{3}$. Elles confortent une hypothèse forte généralement faite lors de l'utilisation de la méthode de l'entrainement, à savoir une similitude des débits de vapeur au sein des poches de cavitation générés pour des fluides différents dans des conditions de fonctionnement identiques.

\section{IV a CONCLUSION}

Des essais expérimentaux ont été réalisés afin d'estimer les débits volumes de la phase vapeur au sein de poches de cavitation. Pour ce faire, des écoulements diphasiques au sein de poches ventilées et cavitantes ont été étudiés sur une même géométrie pour des fluides différents : l'eau et le réfrigérant R114. Ils ont permis d'établir un certain nombre de comportements caractéristiques :

1/ Les poches de cavitations ne sont pas composées de vapeur pure mais d'un mélange diphasique caractérisé par un taux de vide moyen inférieur à $50 \%$.

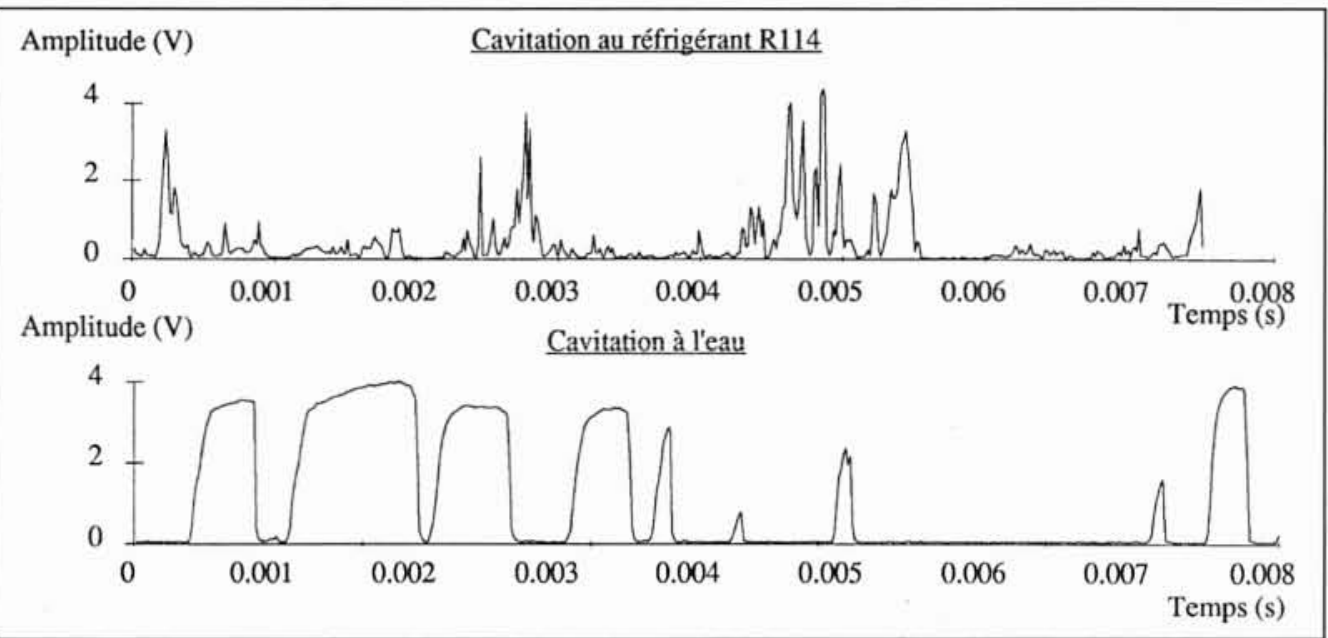

8. Evolution temporelle des signaux issus de la bisonde optique. 
2/ Un écoulement adverse moyen apparaît au voisinage de la paroi sur la partie aval des poches. Son développement est sensible à la géométrie fine de la paroi.

3/ L'amplitude de l'écoulement adverse joue un rôle pondérateur sur le flux de vaporisation au sein des poches.

4/ La méthode de la ventilation permet de donner, en première approximation, un ordre de grandeur intéressant des débits de vapeur au sein des poches de cavitation.

\section{REMERCIEMENTS}

Ce travail a été réalisé dans le cadre de recherches pour le Centre National d'Etudes Spatiales (CNES) et La Société Européenne de Propulsion (SEP). Les différents essais ont été réalisés au Centre de Recherche et d'Essais de Machines Hydrauliques de Grenoble (CREMHyG). Messieurs Eric Janson et Fares Majoub ont été associés à différentes phases de cette étude.

\section{RÉFÉRENCES}

[1] Sturz B. (1996). - Analyse de la structure diphasique et instationnaire de poches de cavitation. Thèse de Doctorat de l'Institut National Polytechnique de Grenoble 1994

[2] Acosta et Perkin discussion avec Holl J.W, Wislicenus G.F. (1961), Scale Effects on Cavitation. Journal of Basic Engineering (1961), vol. 83, pp. 385-398.

[3] Gadd et Grant (1965). - Some Experiments on Cavitaties Behind Disks. J. Fluid Mech. (1965), vol. 23, part 4, pp. 645-656

[4] Billet M.L., Holl J.W., Weir D.S. (1981). - Correlations of Thermodynamic Effets for developped Cavitation. Journal of Fluids Engineering, December 1981, Vol. 103, N4, pp. 534-542
[5] Yoshihara K., kato H., Yamaguchi H., Miyanaga M. (1988). Experimental Study on the Internal Flow of a Sheet Cavity. Cavitation and Multiphase Flow Forum, ASME - FED Vol. 64, pp.94-98

[6] Merle L. (1994), - Etude Expérimentale et Modèle Physique d'un Ecoulement Cavitant avec Effet Thermodynamique. Thèse de Doctorat de l'Institut National Polytechnique de Grenoble 1994

[7] Fruman D.H., Reboud J.L, Stutz B. (1996). - Impact of Vapour Production and Cavity Dynamics on the Estimation of Thermal Effects in Cavitation. Congrès AIRH Hydraulique Machinery and Cavitation, Valencia Septembre 1996

[8] Kueny J.L., Boutet J.M., Jousselin F. (1990), - Analyses d'Images e Cavitation Développée sur un Profil. 3" Coll. Nat. Visualisation et Traitemen d'Images en Mécanique des Fluides, 29 Mai - 10 Juin 1990, Lille France, pp. 256-262

[9] Stutz B., Reboud J.L. (1994), - Experimental Study of the Two Phase Structure of Attached Cavitation. ASME - Cavitation \& Multiphase Flow Forum, Lake Tahoe, Nevada 1994. Fed Vol.194, pp. 41-46

[10] Cartell.jer A., Achard J.L. (1991). - Local Phase Detection Probes in Fluid/Fluid Two-Phase Flows. Rev. Sci. Instrum. 62(2), Feb. 1991 pp. 279-303

[11] Stutz B., Reboud J.L (1996). - Experiments on unsteady cavitation A paraître dans la revue Experiment in Fluids, Jan. 1997. Vol. 22, pp. 191-198.

[12] KAто H. (1984). - Thermodynamic Effect on Incipient and Developed Sheet Cavitation. International Symposium on Cavitation Inception, ASME FED Vol. 16, pp. 127-136.

[13] Delannoy Y., Reboud J.L. (1993). - heat and mass transfert on a vapour cavity. Symposium on Gaz-Liquid flows, Washington, June 20-24 1993. ASME - FED Vol. 165, pp.209-214

[14] Sevik M., ParkS.H. (1973). - The Splitting of Drops and Bubbles by Turbulent Fluid Flow. Journal of Fluids Engineering, March 1973, pp. 53-60.

[15] Bonnin J. (1970). - Début de Cavitation dans Différents Liquides. EDF, Bulletin de la Direction des Etudes et Recherches. Serie A n4, 1970, pp.53-72 